\title{
SCHADEK, Hans, ZOTZ, Thomas, Freiburg (1091-1120), Neue Forschungen zu den Anfängen der Stadt
}

\section{Étienne Champion}

\section{OpenEdition}

\section{Journals}

Édition électronique

URL : http://journals.openedition.org/ifha/1556

DOl : 10.4000/ifha. 1556

ISSN : 2198-8943

Éditeur

IFRA - Institut franco-allemand (sciences historiques et sociales)

Référence électronique

Étienne Champion, «SCHADEK, Hans, ZOTZ, Thomas, Freiburg (1097-1720), Neue Forschungen zu den Anfängen der Stadt », Revue de l'IFHA [En ligne], Date de recension, mis en ligne le 01 janvier 1996, consulté le 22 septembre 2020. URL : http://journals.openedition.org/ifha/1556 ; DOI : https://doi.org/ 10.4000/ifha. 1556

Ce document a été généré automatiquement le 22 septembre 2020.

(C)IFHA 


\title{
SCHADEK, Hans, ZOTZ, Thomas, Freiburg (1091-1120), Neue Forschungen zu den Anfängen der Stadt
}

\author{
Étienne Champion
}

1 Fribourg-en-Brisgau est un objet d'étude passionnant pour le médiéviste par le défi qu'il lance à tout raisonnement déterministe: comment expliquer qu'une situation stratégique aussi favorable, dans un environnement agricole prospère ait attendu le XIIe s. pour connaître le développement d'un véritable centre urbain pérenne. Cette problématique stimule vivement la recherche et donne lieu à une très forte activité éditoriale archéologique et historique dont on présentera ici deux exemples.

2 Destiné aux spécialistes, regroupant historiens et archéologues, le livre de H.S. et T.Z. se consacre aux nouvelles recherches sur les débuts de Fribourg (1091-1120). Le pluriel n'est pas purement rhétorique et se veut en rupture avec la vision traditionnelle d'une ville fondée tardivement et ponctuellement par les Zähringen en 1120. L'ouvrage ne compte ni introduction ni conclusion générale, ce qui limiterait son aspect interdisciplinaire si les auteurs ne montraient dans leur article une véritable connaissance des travaux de chacun. G. FINGERLIN, grâce à l'archéologie, montre comment malgré une forte croissance de l'occupation du sol et de son exploitation depuis l'époque romaine, les anciens centres d'urbanisation ne se sont pas réellement imposés. T.Z., historien, étudie l'occupation du sol et le pouvoir jusqu'à la fondation de la ville, notamment au travers d'une analyse du contrôle de l'eau. Il explique également comment les Zähringen se sont développés entre les centres anciens et non sur leurs sites. Après une introduction qui aurait pu trouver sa place en début d'ouvrage, le chapitre de H. STEUER est essentiellement consacré à une synthèse qui veut replacer le cas de Fribourg dans le cadre général de la ville germanique au XIe s. K. SCHMID, quant à lui, interroge la date de 1091, un autre début de Fribourg, et plus largement les rôles réel et idéalisé des Zähringen. A. ZETTLER se consacre au château, tant par l'étude des textes que par celle des vestiges archéologiques, tandis que M. UNTERMANN présente les apports récents des fouilles urbaines de Fribourg quant au parcellaire, à la 
structures des habitations et à leur occupation. Enfin H.S., élargissant la période, centre son travail sur les bourgeois et la commune.

Le livre dirigé par H.H et H.S. s'adresse assurément à un public plus large. Il n'en reste pas moins d'un haut niveau scientifique, intégrant les apports de la recherche la plus récente. Premier volume d'une série de trois qui couvre histoire de Fribourg jusqu'à nos jours (celui consacré aux XIXe et XXe s. est paru en 1992), cet ouvrage traite la totalité de la période médiévale. Le nombre de collaborateurs est très important, permettant des approches particulièrement diversifiées de l'histoire de la ville. En revanche, l'unité du volume n'est pas évidente et, comme pour l'ouvrage précédent, ni introduction ni conclusion ne permettent de traiter une problématique générale. Le livre présente donc plus une série d'études interdisciplinaire qu'une histoire totale. Outre les premiers chapitres qui reprennent largement les apports de l'ouvrage précédemment recensé et dont on retrouve d'ailleurs certaines cartes et illustrations, on retiendra l'analyse de l'exploitation minière qui, si elle n'est pas une activité proprement citadine, a de fortes répercutions sur l'économie mais aussi sur la politique de la cité. De même, sur le plan institutionnel, sont notamment décrites les implications de l'absence d'évêque sur l'évolution des structures urbaines. L'histoire de l'art occupe également une place de choix, profitant d'une très bonne qualité des reproductions, mais une meilleure intégration dans une problématique historique générale eut été ici particulièrement souhaitable. Ces réserves ne doivent pas faire oublier l'essentiel: cette histoire de Fribourg est un beau livre; il fait honneur au soutien actif que la ville apporte à la recherche sur son passé.

4 Étienne CHAMPION 